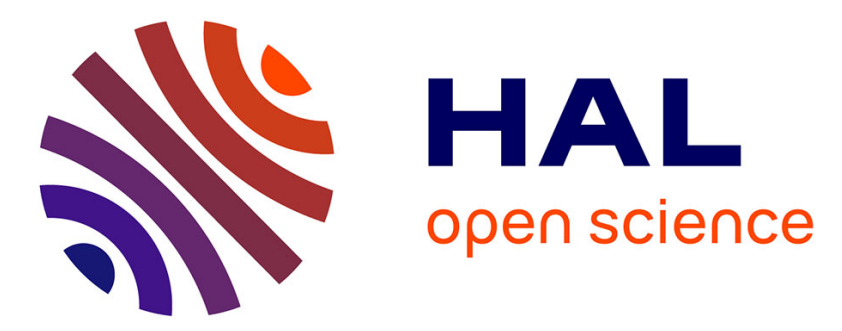

\title{
Control of input redundant affine systems : application to runway axis tracking at landing
}

\author{
Fabrice Villaumé, P Fabre, S.N. Singh, Felix Mora-Camino
}

\section{To cite this version:}

Fabrice Villaumé, P Fabre, S.N. Singh, Felix Mora-Camino. Control of input redundant affine systems : application to runway axis tracking at landing. ACA 2001, 15th IFAC Symposium on Automatic Control in Aerospace, Sep 2001, Bologna, Italy. pp xxxx. hal-01022427

\section{HAL Id: hal-01022427 \\ https://hal-enac.archives-ouvertes.fr/hal-01022427}

Submitted on 10 Jul 2014

HAL is a multi-disciplinary open access archive for the deposit and dissemination of scientific research documents, whether they are published or not. The documents may come from teaching and research institutions in France or abroad, or from public or private research centers.
L'archive ouverte pluridisciplinaire HAL, est destinée au dépôt et à la diffusion de documents scientifiques de niveau recherche, publiés ou non, émanant des établissements d'enseignement et de recherche français ou étrangers, des laboratoires publics ou privés. 


\title{
CONTROL OF INPUT REDUNDANT AFFINE SYSTEMS: APPLICATION TO RUNWAY AXIS TRACKING AT LANDING
}

\author{
F. Villaumé $e^{\star t}$, P. Fabret, \\ S.N. Singh ${ }^{\star}$, F. Mora-Camino* \\ * LAAS-CNRS \\ 7, avenue du Colonel Roche \\ 31077 Toulouse Cedex 04, France \\ + EADS AIRBUS SA \\ 316 , route de Bayonne \\ 31060 Toulouse Cedex 03, France \\ - Dept. Of Electrical and Computer Engineering \\ University of Nevada, Las Vegas \\ NV 89154-4026, USA
}

\begin{abstract}
Among the specific methodologies for the control systems described by nonlinear systems, Nonlinear Dynamic Inversion has gained considerable popularity over the last decade. A complete theory is now available for the design of feedback control laws which transform a nonlinear system into an equivalent linear system. Nevertheless, saturation actuators constraints are not taken into account. In order to solve this drawback, a supervision strategy is proposed in the case of input redundant affine system. Coupled with a generic force and moment nonlinear inverse control laws, this paper shows the viability and efficiency of a such approach.
\end{abstract}

Keywords: Aircraft control, Automatic control, Controls laws, Inverse dynamics control, Nonlinear control, Saturation, Supervision

\section{INTRODUCTION}

The first all weather automatic landing systems were designed during the 60 's and their validation was led through intensive flight tests campaigns. In the following decade, the improved efficiency of guidance systems and on-board computers allowed the final landing phase to be fully automated. Nevertheless, still today important challenges remain, it is for instance the case with the protection against final approach wind-shears. So, the improvement of rollout automatic control systems through the use of advanced control laws is the main issue considered in this communication.

At rollout, the aircraft is submitted not only to atmospheric perturbations (gusts, precipitation) but also to the influence of the runway characteristics (camber and profile, roughness and flatness) and surface contamination (dry, wet, flooded, with or without rubber residue, dry and wet snow, slush, frozen and melting ice). In this situation, the aircraft reactions turn apparent its nonlinear dynamics along its roll and yaw axis. During this phase, the aircraft must perform a safe deceleration while following accurately the runway axis until reaching a feasible runway exit.

This paper displays the results of a study concerned with the development of a non linear inverse dynamics guidance control law for tracking the runway axis during the rollout phase of civil transportation aircraft.

The approach proposed uses a general form of non linear dynamic inversion control coupled to a newly supervision strategy of the actuators available during the automatic landing ground phase. The work presented here incoproporate handling qualities and saturation actuators specifications. The equations of motion are directly manipulated to provide force and moment orders yielding desired responses for runway lateral displacemement and yaw angle, the selected control variable for runway axis tracking. These 
force and moment orders determinated, it is possible to compute, thanks to this newly actuator supervision algorithm, rudder deflection, nose wheel steering angle and dissymetrical braking torque orders in order to satisfy rollout performance specifications and actuators saturation constraints.

The main point of each section, coresponding to the order presented in this paper is 1) an overview of the general theory of non linear dynamic inversion control, 2) the problem description and the mathematical formulation, 3) the manipulation of the aircraft-on-the-ground equations of motion in order to provide the force and moment non linear inverse dynamic control law, 4) the presentation of the actuator supervision algorithm and 5) the simulation results.

\section{GENERAL THEORY OF NONLINEAR DYNAMIC INVERSION}

The objective of this section is to review the inverse control design technique which can be used to shape the dynamics of a non-linear affine system. This technique has been developed mainly by SINGH and RUGH (1972) and FREUND (1973). The affine system is given by :

$$
\begin{aligned}
& \dot{x}=A(x)+B(x) \cdot u \\
& y=C(x)
\end{aligned}
$$

where $\quad A(x) \in M_{n, n}(\Re), \quad B(x) \in M_{n, m}(\Re) \quad$ and $C(x) \in M_{p n}(\Re)$. Vector $x$ is a state vector of dimension $n$, vector $u$ is an input vector of dimension $m$ and vector $y$ is an output vector of dimension $p$. It is supposed that $x$ and $u$ are subject to saturation constraints such that :

$$
\left\{\begin{array}{l}
x_{\min } \leq x \leq x_{\max } \\
u_{\min } \leq u \leq u_{\max }
\end{array}\right.
$$

The relative degrees of the outputs of system (1-2) are obtained by differentiating the dynamics of each output until some components of the input vector appear explicitly. Since at most $p$ independent outputs must be controlled from $m$ different inputs, it is assumed that $\operatorname{dim}(y)=p \leq \operatorname{dim}(u)=m$. Introducing the $\mathrm{k}^{\text {th }}$-order differentiation operator, $L_{A}^{k}($.$) , such that$

$$
\left\{\begin{array}{l}
L_{A}^{k}(x)=\left[\frac{\partial}{\partial x} L_{A}^{k-1}(x)\right] \cdot A(x) \\
L_{A}^{0}(x)=x
\end{array}\right.
$$

the successive derivatives of the $\mathrm{i}^{\text {th }}$ component of $y$ are given by :

$$
\begin{aligned}
& \dot{y}_{i}=C_{i} \dot{x}=C_{i} A(x)+C_{i} B(x) u=C_{i} L_{\lambda}^{\prime}(x) \\
& \ddot{y}_{i}=C_{i} \ddot{x}=C_{i}\left[\frac{\partial}{\partial x} L_{A}^{l}(x)\right] A(x)+C_{i}\left[\frac{\partial}{\partial x} L_{A}^{I}(x)\right] B(x), u=C_{i} L_{L_{A}}^{2}(x) \\
& y_{i}^{\left(d_{i}\right)}=C_{i} x^{\left(d_{i}\right)}=C_{i}\left[\frac{\partial}{\partial x} I_{A}^{d_{i}-t}(x)\right] A(x)+C_{i}\left[\frac{\partial}{\partial x} L_{A}^{d_{1}-t}(x)\right] B(x) \cdot u
\end{aligned}
$$

where $d_{i}$ is the order of the time derivative of $y_{i}$ such that :

$$
C_{i}\left[\frac{\partial}{\partial x} L_{A}^{d_{i}-1}(x)\right] \cdot B(x) u \neq 0(5)
$$

In the full paper the concept of relative degree is refined in the context of input redundancy so that input saturation situations can be tackled with the non-linear inverse control technique. Once the relative degrees have been determined, the output dynamics can be represented as,

$$
y^{(d)}=\left[\begin{array}{c}
y_{1}^{\left(d_{1}\right)} \\
y_{2}^{\left(d_{2}\right)} \\
\vdots \\
y_{m}\left(d_{m}\right)
\end{array}\right]=\left[\begin{array}{c}
C_{1} L_{A}^{d_{1}}(x) \\
C_{2} L_{A}^{d_{3}}(x) \\
\vdots \\
C_{m} L_{A}^{d_{m}}(x)
\end{array}\right]+\left[\begin{array}{c}
C_{1} \frac{\partial}{\partial x} L_{A}^{d_{z}-1}(x) \\
C_{2} \frac{\partial}{\partial x} L_{A}^{d_{2}-I^{\prime}}(x) \\
\vdots \\
C_{m} \frac{\partial}{\partial x} L_{A}^{d_{m}-1}(x)
\end{array}\right] \cdot B(x) \cdot u
$$

or in a global affine form :

$$
y^{(d)}=A^{*}(x)+B^{*}(x) \cdot u
$$

When no state or control saturation constraints are considered, a sufficient condition for the existence of an inverse system model to (1) is that $B^{*}$ in (6) be non-singular (SINGH and RUGH, 1972; FREUND, 1973). If this is the case, then, choosing a $d_{i}^{\text {th }}$ order linear dynamics for the $\mathrm{i}^{\text {th }}$ output of the system, the whole system follows the equations :

$$
\begin{gathered}
\dot{x}=A(x)-B(x)\left[B^{*}(x)\right]^{-1} A^{*}(x)+B(x)\left[B^{*}(x)\right]^{-1} y^{(d)} \\
u=-\left[B^{*}(x)\right]^{-1} A^{*}(x)+\left[B^{*}(x)\right]^{-1} y^{(d)}
\end{gathered}
$$

Then, if the outputs and their respective dynamics have been chosen adequately, the inner dynamics can be made stable and the outputs of the system follow their desired trajectories.

\section{PROBLEM DESCRIPTION AND MATHEMATICAL FORMULATION}

\subsection{Objectives}

The main characteristics of the IIIb landing Category is the absence of decision height. In this case, a failure of the Automatic Pilot (AP) between 100 feet and the stop on the runway is a catastrophic risk whose probability must be less than $10^{-5}$, while in manual mode, the control of the aircraft on the 
runway for a speed above 50 knots needs good outside visual references. For the IIIa landing Category these guarantees must be fulfilled until touchdown where the pilot has to disconnect the Auto-Pilot (AP).

The Automatic Rollout Function guides the aircraft over the runway during the deceleration. The airworthiness regulations fix the lateral tolerances that must be satisfied by the aircraft along the runway axis. According to the Federal Aviation Authorities, the probability to leave the $\pm 70 \mathrm{ft}$ safe zone with respect to the runway axis must be less than $10^{-6}$.

Until today, the guidance references are provided to the aircraft by the ILS (Instrument Landing System), set on the runway axe, and heading, altitude, speed measurement data while the active control devices are the rudder and the nose wheel.

The originality of the proposed Rollout Control Law is the simultaneous control and supervision of the available actuators, the nose wheel orientation, the rudder deflection and the dissymmetrical braking, through the use of an advanced non-linear control technique : Nonlinear Dynamic Inversion Control. The main objectives are here to correct the initial deviation at touchdown and to improve robustness of the runway axis tracking with respect to different perturbations (winds, runway surface state, ILS signal noises).

\subsection{Modeling of ground motion}

Thanks to the particular form of the flight mechanics equations and the tire/runway contact relations, the ground motion equations of an aircraft can be decomposed into its longitudinal and lateral components. If longitudinal ground dynamics are mainly independent from the lateral dynamics, these latter are parametrized by the longitudinal speed of the aircraft. Then, the lateral ground dynamics can then be represented by a set of equations such as :

$$
\dot{x}=f\left(\underline{x}, V_{x}\right)+g\left(\underline{x}, V_{x}\right) \underline{u}
$$

with $\underline{x}=(\beta, r, \psi, y)^{T}$ and $\underline{u}=(\delta r, \delta e, \delta f)^{T}$, where $\beta$ is the side slip angle, $r$ is the yaw rate, $\psi$ is the heading angle, $y$ is the lateral deviation from the runway axis, $V_{x}$ is the longitudinal ground speed, $\delta r$ is the rudder deflection, $\delta e$ is the nose wheel steering angle and $\delta f$ is the dissymmetrical braking torque.

The output vector of interest is here: $\underline{Y}=(y, r)^{T}$. The equation (10) is nonlinear affine, which justify the use of the nonlinear dynamic inversion control methodology.
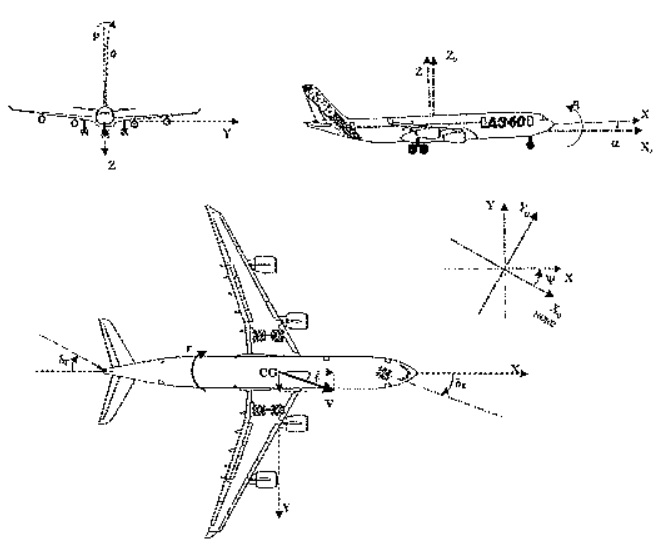

Fig. 1. Sign convention uses in the modeling of aircraft ground motion

\section{THE NONLINEAR INVERSE CONTROL LAW FOR RUNWAY AXIS TRACKING}

\subsection{Inner-loop control law}

Here the relative degrees of the chosen outputs are two in both cases, so that two second order dynamics can be settled according to the non-linear inverse control approach :

$$
\underline{Y}^{(2)}=\left(\begin{array}{c}
-2 z_{y} \omega_{y} \dot{y}-\omega_{y}^{2} y \\
-2 z_{\psi} \omega_{\psi} \dot{\psi}-\omega_{\psi}^{2} \psi
\end{array}\right)=\left(\begin{array}{c}
\dot{V}_{y} \\
\dot{r}
\end{array}\right)
$$

where $V_{y}=V_{x} \cdot(\psi+\beta)$ and $\dot{\psi}=r . V_{y}$ corresponds to the lateral displacement rate. Then, it is possible to express the second order derivatives of the control variables with respect to the state variables:

$$
\left(\begin{array}{c}
\dot{V}_{y} \\
\dot{r}
\end{array}\right)=\left(\begin{array}{cccc}
V_{x} & 0 & 0 & 0 \\
0 & 1 & 0 & 0
\end{array}\right) \dot{\underline{x}}+\left(\begin{array}{cccc}
\dot{V}_{x} & V_{x} & \dot{V}_{x} & 0 \\
0 & 0 & 0 & 0
\end{array}\right) \underline{x}
$$

Then the whole set of inputs must satisfy conditions which can be written synthetically as :

$$
A(\underline{x}) \underline{u}=\underline{g}(\underline{x}, \underline{\lambda})
$$

where $A(x) \in M_{2,3}(\Re), \underline{u}=(\delta r, \delta e, \delta f)^{T}$ and $\underline{\lambda}$ gathers the tuning parameters $\left(z_{y}, \omega_{y}\right)$ and $\left(z_{\psi}, \omega_{\psi}\right)$ of the rollout control law.

At this point the need for a supervisor which determines dynamically the current active actuators necessary to fulfill efficiently the tracking function (around references $y_{c}=0$ and $\psi_{c}=0$ ), appears necessary. 


\subsection{Supervision strategy}

The main idea of this strategy is to maintain active, whenever possible, the control inputs used in the current rollout laws. The dissymmetrical braking is until today used only in manual mode and it appears interesting here to introduce it when at least one of the two main actuators reaches saturation. Following this approach, equation (13) provides the corresponding input $\underline{u}$ according to $\underline{x}$ and $\underline{\lambda}$. Then, the reference values for the braking torque, which must be applied to the left and right main landing gears, can be determined.

Thanks to this consideration, it is possible to design a supervision strategy in order to compute the actuator orders in accordance with the current aircraft states and with the physical limits of the actuators. In writing aircraft-on-the-ground equations, the natural control inputs are the yaw torque $M_{c c t}$ and the lateral force $F_{a c t}$ generated by the actuators. Their expression according to the traditional control actuator deflections is such as :

$$
\left(\begin{array}{c}
F_{a c t} \\
M_{a c t}
\end{array}\right)=\left(\begin{array}{lll}
\mu_{1}\left(V_{x}\right) & \mu_{2}\left(V_{x}\right) & \mu_{3}\left(V_{x}\right) \\
\mu_{4}\left(V_{x}\right) & \mu_{5}\left(V_{x}\right) & \mu_{6}\left(V_{x}\right)
\end{array}\right)\left(\begin{array}{l}
\delta r \\
\delta e \\
\delta f
\end{array}\right) \text { (14) }
$$

The equation (14) can be written as follows :

$$
\left(\begin{array}{c}
F_{a c t} \\
M_{a c t}
\end{array}\right)=\left(\begin{array}{ll}
\mu_{I}\left(V_{x}\right) & \mu_{2}\left(V_{x}\right) \\
\mu_{4}\left(V_{x}\right) & \mu_{5}\left(V_{x}\right)
\end{array}\right)\left(\begin{array}{l}
\delta r \\
\delta e
\end{array}\right)+\left(\begin{array}{l}
\mu_{3}\left(V_{x}\right) \\
\mu_{6}\left(V_{x}\right)
\end{array}\right) \cdot \delta f
$$

in order to distinguish the traditional actuators (rudder and nose wheel) to the dissymetrical braking which will be used automatically when they are about to reach saturation constraints. Then, the relation (15) gives :

$$
\left(\begin{array}{l}
\delta r \\
\delta e
\end{array}\right)=\left(\begin{array}{ll}
\mu_{l}\left(V_{x}\right) & \mu_{2}\left(V_{x}\right) \\
\mu_{f}\left(V_{x}\right) & \mu_{5}\left(V_{x}\right)
\end{array}\right)^{-1}\left[\left(\begin{array}{c}
F_{a c t} \\
M_{w c t}
\end{array}\right)-\left(\begin{array}{l}
\mu_{3}\left(V_{x}\right) \\
\mu_{6}\left(V_{x}\right)
\end{array}\right) \delta f\right]
$$

which can be simplified as follows :

$$
\left\{\begin{array}{l}
\delta r=\gamma_{1}\left(V_{x}, F_{a c t}, M_{a c t}\right)+\gamma_{2}\left(V_{x}, F_{a c t}, M_{a c t}\right) . \delta f \\
\delta e=\gamma_{3}\left(V_{x}, F_{a c t}, M_{a c t}\right)+\gamma_{4}\left(V_{x}, F_{a c t}, M_{a c t}\right) \delta f
\end{array}\right.
$$

To satisfy the rudder and nose wheel steering deflection constraints, it is possible to compute $\delta f_{m i n}^{\delta r}$, $\delta f_{\max }^{\delta r}, \delta f_{\min }^{\delta e}$ and $\delta f_{\text {max }}^{\delta e}$ such that :

$$
\begin{aligned}
& \delta f_{\min }^{\delta r} \leq \delta f \leq \delta f_{\max }^{\delta r} \\
& \delta f_{\min }^{\dot{\delta}} \leq \delta f \leq \delta f_{\max }^{\dot{\alpha u}}
\end{aligned}
$$

and, simultaneously,

$$
\delta f_{\min } \leq \delta f \leq \delta f_{\max }
$$

Then, two new limits for the dissymetrical braking torque can be introduced :

$$
\left.\begin{array}{l}
\Delta f^{+}=\min \left\{\delta f_{\max }, \delta f_{\max }^{\delta \cdot}, \delta f_{\max }^{\delta e}\right. \\
\Delta f^{-}=\max \left\{\delta f_{\min }, \delta f_{\min }^{\delta \cdot}, \delta f_{\min }^{\delta e}\right.
\end{array}\right\}
$$

Once, the condition $\Delta f^{-} \leq \Delta f^{+}$is checked, three possible situations can appear:

$$
\begin{aligned}
& \text { - if } \Delta f^{-} \geq 0, \text { then } \delta f=\Delta f^{-}, \\
& \text {- if } \Delta f^{-} \leq 0 \leq \Delta f^{+}, \text {then } \delta f=0, \\
& \text { - if } \Delta f^{+} \leq 0 \text {, then } \delta f=\Delta f^{+},
\end{aligned}
$$

The above algorithm provides a strategy to organize in an efficient way the activation of the available actuators.

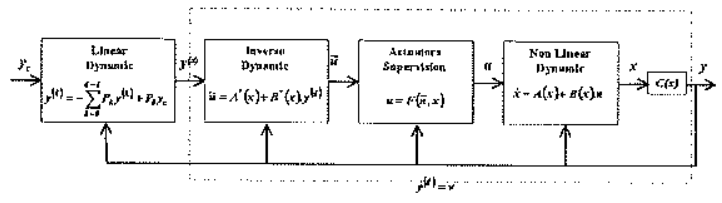

Fig. 2. General block diagram of the proposed solution

\section{ANALYSIS OF THE RESULTING SUPERVISED NONLINEAR CONTROL LAW}

In order to analyze the closed-loop system's behavior and eventually refine the high level linear controller tuning, time response are really useful. Simulations are performed and shown that closed-loop system has satisfactory performance in spite of the saturation of the traditional aircraft-on-the ground actuators. Moreover, the results shown that with the use of the above saturation actuators supervision coupled with the Nonlinear Dynamic Inversion method, the aircraft's response is almost identical to the prescribed dynamics.

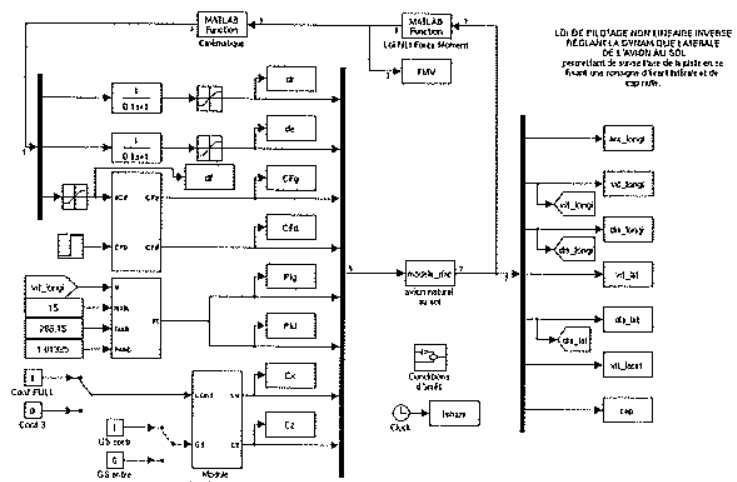

Fig.3. Closed-loop aircraft Simulink ${ }^{(}$simulation chart 
Successively, three charts are presented. The first one displays the Simulink ${ }^{\odot}$ block diagram used to simulate the closed-loop aircraft (Fig.3.). The following chart presents the time responses of aircraft states and actuators orders with a standard force and moment nonlinear dynamic inversion control law for the runway axis tracking (Fig.4.)
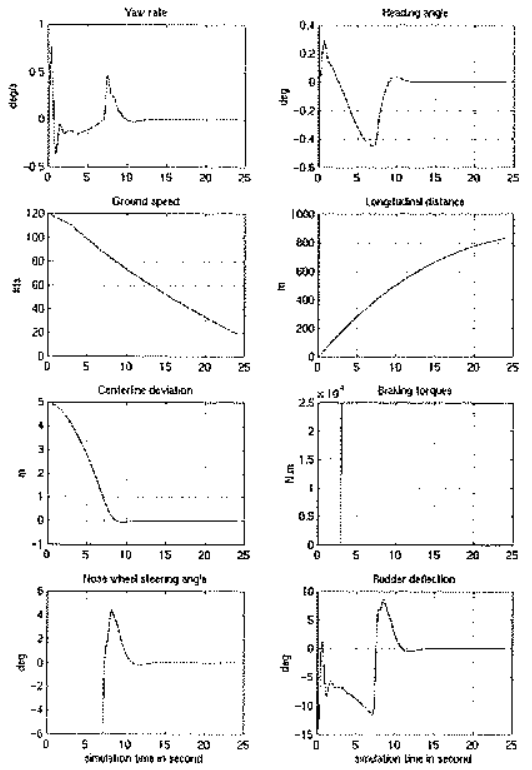

Fig.4. Time responses without differential braking supervision

The last chart highlights the advantage of the differential braking supervision algorithm when one of the traditional actuator saturates (Fig.5.). The dashed line presents differential braking order.
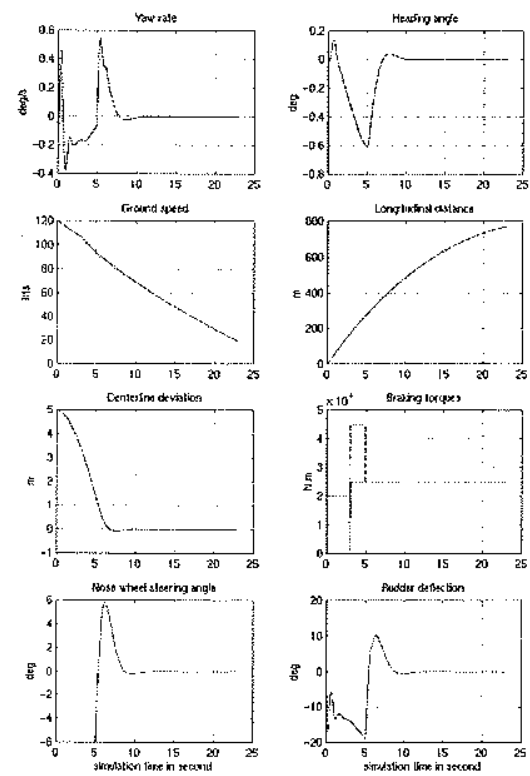

Fig.5. Time responses with differential braking supervision

\section{CONCLUSION}

The study briefly reported in this paper is devoted to the development of a new device for improved lateral rollout control for wide body civil transportation aircraft. A tentative structure based on Nonlinear Dynamic Inversion Control theory has been proposed. Moreover, Dynamic Inversion is an attractive technique because of:

- the use of dynamic models and fullstate feedback to globally linearize dynamics of selected controlled variables,

- the simplicity of the controller structure which avoids gain schedules,

The absence of unobservable modes in the runway axis tracking problem makes easier the application of Dynamic Inversion Control theory. Besides, the actuator saturation problem is originally solved thanks to the introduction of a supervision algorithm coupled with an adapted nonlinear dynamic inversion control law. A quicker runway axis tracking is also obtained.

The results obtained with this approach are very stimulating. Nevertheless, before this kind of system could be effectively implemented onboard modern aircraft, either as rollout director for the pilot or as a fully automated function, extensive evaluation studies, involving not only numerical simulations but also ground tests, should be performed.

\section{REFERENCES}

BARNES, A.G. and YAGER, T.J. (1985) Simulation of aircraft on and close to the ground AGARDDograph AG 285

DUGOFF, H. (1969) - Tire performance characteristics affecting vehicle aircraft response to steering and braking control inputs - NTIS PB - 187 667

FREUND, E. (1973) - Decoupling and pole assignment in non linear systems - Electron. Lett., 9, 373-374

MORA CAMINO, F. (1993) - Systèmes de conduite automatique et de gestion du vol-ENAC

RODNEY HANKE, C. (1971) - The simulation of a large jet transport aircraft; Vol 1. Mathematical model - NASA CR 1756

SINGH, S.N. and RUGH, W.J. (1972) - Decoupling in a class of non-linear systems by state variable feedback - AIAA J. Dynamics Syst. Meas. Control, 323-329 